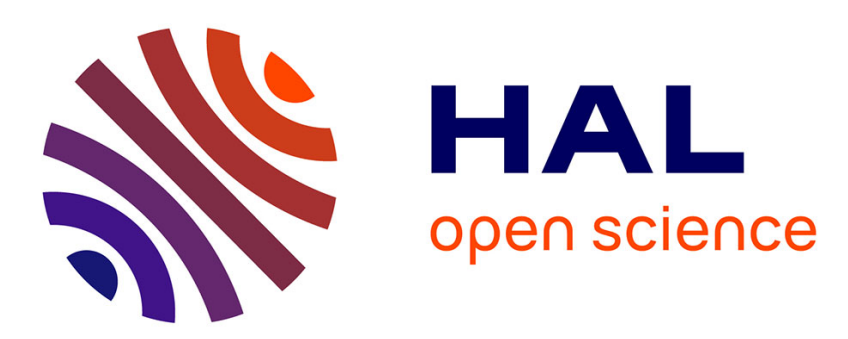

\title{
Réaménagements territoriaux, contrôle des populations et stratégies contre-insurrectionnelles \\ Pamela Colombo
}

\section{To cite this version:}

Pamela Colombo. Réaménagements territoriaux, contrôle des populations et stratégies contreinsurrectionnelles. Critique Internationale, 2018, Les villages stratégiques: politiques contreinsurrectionnelles et regroupements de populations, 2018/2 (79), pp.9- 24. hal-02127785

\section{HAL Id: hal-02127785 \\ https://hal.science/hal-02127785}

Submitted on 14 May 2019

HAL is a multi-disciplinary open access archive for the deposit and dissemination of scientific research documents, whether they are published or not. The documents may come from teaching and research institutions in France or abroad, or from public or private research centers.
L'archive ouverte pluridisciplinaire HAL, est destinée au dépôt et à la diffusion de documents scientifiques de niveau recherche, publiés ou non, émanant des établissements d'enseignement et de recherche français ou étrangers, des laboratoires publics ou privés.

\section{(ㅇ)(1) $\$$}

Distributed under a Creative Commons Attribution - NonCommercial - NoDerivatives| 4.0 
The research leading to these results has received funding for the European Union's Horizon 2020 (H2020-MSCA-IF-2014-GF) research and innovation programme under the Marie Skłodowska-Curie grant agreement No 654923

Les recherches sur lesquelles a pris appui cette publication ont fait l'objet d'un financement du programme de recherche et d'innovation Horizon 2020 (H2020-MSCAIF-2014-GF) de l'Union européenne dans le cadre de la convention Marie Skłodowska-

Curie $n^{\circ} 654923$.

Pamela COLOMBO

Marie Skłodowska-Curie Research Fellow. IRIS (Institut de Recherche Interdisciplinaire sur les enjeux Sociaux), EHESS (École des Hautes Études en Sciences Sociales), Paris.

pamela.colombo@ehess.fr

Colombo, P. (2018). "Réaménagements territoriaux, contrôle des populations et stratégies contre-insurrectionnelles », in Critique International, No79, avril-juin 2018. France. pp.9- 24

\section{Réaménagements territoriaux, contrôle des populations et strategies contre- insurrectionnelles}

par Pamela Colombo

Depuis le début de la guerre froide, les politiques de contre-insurrection ont eu pour objectif non seulement de détruire les mouvements de guérilla, mais aussi de " gagner les cœurs et les esprits $»^{1}$ de la population. Parmi les différentes techniques utilisées pour poursuivre cet objectif, on en retrouve une qui se reproduit depuis bientôt sept décennies : la création de villages ex nihilo. Bien que ces programmes aient reçu différentes dénominations, nous proposons de les rassembler sous le concept de villages stratégiques $^{2}$. Ces villages ont pour objectif de couper les liens entre la population et les mouvements guérilleros par le déplacement et le regroupement des populations rurales et autochtones, et d'utiliser l'urbanisation forcée comme un outil de contre-insurrection

\footnotetext{
${ }^{1}$ L'idée de « gagner les cœurs et les esprits » est appliquée pour la première fois dans un contexte de contre-insurrection en Malaisie à la fin des années 1950 et est attribuée au général britannique Sir Gerald Templer. Robert Egnell, "Winning "Hearts and Minds"? A Critical Analysis of Counter-Insurgency Operations in Afghanistan », Civil Wars, 12 (3), 2010, p. 282-303. Voir aussi Christian Olsson, « Guerre totale et/ou force minimale ? Histoire et paradoxes des "cœurs et des esprits" », Cultures \& Conflits, 67, 2009, p. 35-62.

2 Il faut bien souligner que les historiens Nicole Sackley et Christian Gerlach ont déjà proposé des généalogies utilisant aussi le terme en anglais de « strategic village ». Nicole Sackley, « The village as Cold War sites: experts, development, and the history of rural reconstruction », Journal of Global History, 6, 2011, pp.481-504. ; Christian Gerlach Extremely Violent Societies. Mass Violence in the Twentieth-Century World, Cambridge, Cambridge University Press, 2010.
} 
permettant de mieux contrôler les habitants, de gagner des territoires et d'élargir (ou de consolider) le pouvoir de l'État sur ces zones. Quelles que soient les différentes applications de cette politique à travers le monde, on constate souvent que la construction des villages stratégiques implique le soutien de gouvernements étrangers, la création de milices (souvent avec la population déplacée), la destruction des anciens espaces de vie ruraux $^{3}$, mais aussi de profonds changements dans le territoire ainsi que l'introduction de nouvelles infrastructures. Ce dossier est le premier ouvrage qui rassemble des travaux de recherche sur leurs différentes mises en œuvre des villages stratégiques dans le monde.

Nous commencerons d'abord par expliquer les éléments constitutifs de ce nouvel objet d'étude, ensuite nous étudierons la circulation transnationale des savoir-faire militaires et leurs adaptations locales, mais aussi les éléments contre-insurrectionnels qui font de ces regroupements de populations un cas singulier. Nous explorerons également la possibilité de penser ces villages comme des espaces urbains utilisés comme des instruments de guerre. Enfin, nous examinerons d'autres éléments constitutifs : le rôle des politiques de développement et les doctrines de sécurité nationale, les régimes de pouvoir que le travers et le constitue tant comme dispositif disciplinaire que sécuritaire, la fonction des villages stratégiques dans des programmes économiques d'expansion capitaliste et la façon dont ce type de technique militaire permet d'analyser le changement d'échelle dans les façons de faire la guerre.

Les « villages stratégiques » : un objet d'étude nouveau

Des villages stratégiques ont été créés en Afrique et en Asie, notamment dans des contextes de guerres de décolonisation et avec l'appui des puissances coloniales: la Grande-Bretagne a construit des New Villages en Malaisie ${ }^{4}$, au Kenya ${ }^{5}$ et en Inde ${ }^{6}$, les États-Unis ont édifié des Strategic Hamlets au Vietnam ${ }^{7}$, le Portugal a créé des aldeamentos en Angola et au Mozambique ${ }^{8}$, et la France a bâti des «villages

\footnotetext{
${ }^{3}$ Jim Glassman, « Counter-Insurgency, Ecocide and the Production of Refugies », Refuge, 12 (1), 1992, p. 27-30.

4 Karl Hack, "Malaya - Between Two Terrors: "People's History" and the Malayan Emergency ", dans Hannah Gurman (ed.), Hearts and Minds. A People's History of Counterinsurgency, New York/Londres, The New Press, 2013, p. 17-49.

${ }^{5}$ Daniel Branch, Defeating Mau Mau, Creating Kenya: Counterinsurgency, Civil War, and Decolonization, Cambridge, Cambridge University Press, 2009.

${ }^{6}$ Nicole Sackley, « Village Models: Etawah, India, and the Making and Remaking of Development in the Early Cold War », Diplomatic History, 37 (4), 2013, p. 749-778

7 Élie Tenenbaum, «Les déplacements de populations comme outil de contre-insurrection: l'exemple du programme des hameaux stratégiques au sud-Vietnam », Guerres mondiales et conflits contemporains, 3 (239), 2010, p. 119-141.

${ }^{8}$ João Paulo Borges Coelho, «Protected Villages and Communal Villages in the Mozambican Province of Tete (1968-1982): A History of State Resettlement Policies, Development and War », Doctoral dissertation, University of Bradford, 1993.
} 
d'autodéfense » en Indochine ${ }^{9}$ et des « centres de regroupement » au Cameroun ${ }^{10}$ et en Algérie $^{11}$. Il y a eu d'autres cas où l'influence directe de gouvernements étrangers est moins évidente : c'est le cas des Protected Villages créés en République de la Rhodésie du Sud (Zimbabwe) ${ }^{12}$. Dans un tout autre cadre que celui des guerres postcoloniales, alors que des régimes autoritaires s'instauraient en Amérique latine durant les années 1970 et 1980, des villages stratégiques ont été construits aussi en Argentine ${ }^{13}$, au Brésil ${ }^{14}$, au Pérou $^{15}$, au Salvador ${ }^{16}$ et au Guatemala ${ }^{17}$. Le rôle des gouvernements étrangers dans ces cas est plus indirect et se fait souvent sous couvert d'aide au développement ${ }^{18}$. Loin d'avoir disparu, ces programmes semblent être encore utilisés dans des scénarios de guerres contemporaines. Quelques auteurs ont signalé des similarités entre les villages stratégiques et les programmes de relocalisation planifiée du gouvernement turc au Kurdistan $^{19}$. De plus, la possibilité a été explorée qu'il existe des similitudes entre les

${ }^{9}$ C. Gerlach, Extremely Violent Societies. Mass Violence in the Twentieth-Century World, op. cit. ; Fabien Sacriste, « Les camps de "regroupement". Une histoire de l'État colonial et de la société rurale pendant la guerre d'indépendance algérienne (1954-1962)», thèse en histoire, FRAMESPA - France Méridionale et Espagne, 2014, p. 143.

${ }^{10}$ F. Sacriste, «Les camps de "regroupement". Une histoire de l'État colonial et de la société rurale pendant la guerre d'indépendance algérienne (1954-1962)», cité; Thomas Deltombe, Manuel Domergue, Jacob Tatsitsa, Kamerun!: une guerre cachée aux origines de la Françafrique (1948-1971), Paris, La Découverte, 2011.

${ }^{11}$ Mahfoud Bennoune, «La doctrine contre-révolutionnaire de la France et la paysannerie algérienne : les camps de regroupement (1954-1962) », Nord-Sud, 14, 2001, p. 57. ; F. Sacriste, «Les camps de "regroupement". Une histoire de l'État colonial et de la société rurale pendant la guerre d'indépendance algérienne (1954-1962) », cité ; Sylvie Thénault, Violence ordinaire dans l'Algérie coloniale. Camps, internements, assignation à résidence, Paris, Odile Jacob, 2012.

${ }^{12} \mathrm{C}$. Gerlach, Extremely Violent Societies. Mass Violence in the Twentieth-Century World, op. cit.

${ }^{13}$ Sur le cas argentin, voir Pamela Colombo, «L'urbanisation forcée comme politique contreinsurrectionnelle. La vie au sein des villages stratégiques construits en Argentine (1976-1978) », Cultures \& Conflits, 103-104, 2016, p. 91-110.

${ }^{14}$ Sur le Brésil, voir Paulo Tavares, « La naturaleza política de la selva: una arqueología botánica del genocidio ", Revista Clepsidra (dossier Políticas urbanas en contextos de violencia de Estado en América Latina), 1, 2018 ; Renato Leao Rego, "Comunidades planejadas na Amazônia: o Urbanismo Rural e a utopia de uma nova civilização", Actas del XIV Coloquio Internacional de Geocritica. Barcelona: Universitat de Barcelona. Recuperado em, 25, 2016, pp.1055-1064.

${ }^{15}$ Sur le Pérou, voir Lewis Taylor, « Counter-Insurgency Strategy, the PCP-Sendero Luminoso and the Civil War in Peru, 1980-1996», Bulletin of Latin American Research, 17 (1), 1998, p. 35 58.

${ }^{16}$ Sur le Salvador, voir N. Sackley, « The village as Cold War sites: experts, development, and the history of rural reconstruction », art. cité.

${ }^{17}$ Sur le Guatemala : Finn Stepputat, «Politics of Displacement in Guatemala », Journal of Historical Sociology, 12 (1), 1999, p. 54-80 ; Forensic Architecture Group, « The Earth Scorched: Environmental Violence and Genocide in the Ixil Triangle, Guatemala, 1980-1983 », dans Forensis. The Architecture of Public Truth, Berlin, Sternberg Press et Forensic Architecture Group, 2014.

${ }^{18}$ Notamment dans les cas du Guatemala et du Salvador, pour lesquels il a été signalé qu'ils ont reçu un financement de la part de l'USAID pour aider à construire les villages. N. Sackley, «The village as Cold War sites: experts, development, and the history of rural reconstruction », art. cité, p. 502. ${ }^{19}$ Joost Jongerden, « Village Evacuation and Reconstruction in Kurdistan (1993-2002) », Études rurales, dossier Ruralité, urbanité et violence au Kurdistan, 186, 2010, p. 77-100. 
villages stratégiques et les programmes mis en place en Irak et en Afghanistan ${ }^{20}$ dans le cadre de la guerre contre $«$ le terrorisme $»^{21}$.

Nous considérons les villages stratégiques comme des espaces hybrides situés entre le monde civil et le monde militaire, des espèces de cristallisations socio-spatiales de situations de $"$ ni guerre ni paix $»^{22}$. Dans certains cas, ces villages perdurent encore aujourd'hui et les personnes qui y ont été déplacées de force continuent à y vivre (par exemple en Argentine, au Guatemala ou en Malaisie). Dans d'autres cas, les villages n'ont pas persisté, du fait de sabotages ou de l'inefficacité des constructions et des infrastructures (par exemple au Vietnam). Malgré la mise en œuvre récurrente de ces stratégies militaires durant plus de sept décennies et dans le monde entier, la question n'a été jusqu'à présent que sporadiquement et marginalement étudiée. Seuls quelques historiens ${ }^{23}$, géographes ${ }^{24}$, politistes ${ }^{25}$ et $\operatorname{architectes}^{26}$ ont accordé un intérêt à ces espaces singuliers et pourtant centraux pour la compréhension de ces techniques étatiques de réorganisation et de contrôle des populations et territoires « marginaux $»^{27}$.

C'est dans le but de pallier cette lacune de la littérature scientifique que ce dossier de Critique internationale cherche à mener une discussion interdisciplinaire et à ouvrir un débat autour de la mise en place globale des villages stratégiques. L'une de nos ambitions est de mieux comprendre ces programmes, non seulement en donnant à voir l'histoire de

${ }^{20}$ Oliver Belcher, «The Afterlives of Counterinsurgency: Post-colonialism, Military Social Science, and Afghanistan 2006-2012 », PhD thesis, The University of British Columbia, 2013 ; É. Tenenbaum, « Les déplacements de populations comme outil de contre-insurrection: l'exemple du programme des hameaux stratégiques au sud-Vietnam », art. cité.

${ }^{21}$ Dans l'état actuel de notre recherche, nous sommes loin de pouvoir fournir une liste définitive des expériences de villages stratégiques. Il est important de souligner que les cas réunis ici ne représentent pas la totalité, mais rassemblent seulement les cas qui ont été travaillés par d'autres chercheurs ou par nous-mêmes. Des auteurs comme J. P. Borges Coelho (voir l'article dans ce dossier) signalent d'autres cas africains, comme ceux de l'Éthiopie et de l'Afrique du Sud-Ouest (Namibie). De plus, C. Gerlach signale les cas de la Guinée-Bissau, de la Thaïlande, du Timor oriental et du Bangladesh. C. Gerlach, Extremely Violent Societies. Mass Violence in the Twentieth-Century World, op. cit.

${ }^{22}$ Dominique Linhardt, Cédric Moreau de Bellaing, « Ni guerre, ni paix. Dislocations de l'ordre politique et décantonnements de la guerre », Politix, 104 (4), 2013, p. 7-23.

${ }^{23}$ N. Sackley, "The village as Cold War sites: experts, development, and the history of rural reconstruction », art. cité ; F. Sacriste, «Les camps de "regroupement". Une histoire de l'État colonial et de la société rurale pendant la guerre d'indépendance algérienne (1954-1962) », cité.

${ }^{24}$ James Tyner, « Biopower in Vietnam », dans J. Tyner, War, Violence, and Population. Making the Body Count, New York, The Guilford Press, 2009; O. Belcher, "The Afterlives of Counterinsurgency: Post-colonialism, Military Social Science, and Afghanistan 2006-2012», cité.

${ }^{25}$ É. Tenenbaum, «Les déplacements de populations comme outil de contre-insurrection: l'exemple du programme des hameaux stratégiques au sud-Vietnam », art. cité ; F. Stepputat, «Politics of Displacement in Guatemala », art. cité.

${ }^{26}$ Forensic Architecture Group, « The Earth Scorched: Environmental Violence and Genocide in the Ixil Triangle, Guatemala, 1980-1983 » cité.

${ }^{27}$ Ainsi, il existe des écrits militaires qui s'intéressent plutôt à la planification et à la mise en œuvre des villages stratégiques pour en mesurer le « succès » ou l' " échec » d'un strict point de vue militaire. David Galula, Contre-insurrection. Théorie et pratique, Paris, Économica, [1964] 2008 ; Robert Thompson, Defeating Communist Insurgency: The Lessons of Malaya and Vietnam, F. A Praeger, 1966 ; Roger Trinquier, La guerre moderne, Paris, Économica, (1961) 2008. 
leur déploiement, mais aussi en essayant de saisir leurs différents devenirs sociaux, politiques, économiques et militaires. Les auteurs réunis ici explorent également les discours des acteurs impliqués dans ces programmes, en se focalisant sur les acteurs étatiques (notamment les militaires) et sur la population directement affectée par ces politiques. Cependant, même si la réalisation de ce type de projet a été confiée dans la grande partie des cas à l'armée, des acteurs civils ont eux aussi joué un rôle important (instituts de logement social, architectes, planificateurs urbains...). Signalons enfin que la plupart de ces villages se situent dans des zones rurales, et que leur design était géométriquement conçu pour permettre une surveillance rapide et efficace de la population à travers des éléments tels que des tours contrôle, des postes de vérification pour accéder aux villages, de nouvelles routes, des réseaux barbelés, voire des murs séparant le village de l'extérieur. Un contrôle était exercé sur la mobilité, et sur les façons d'habiter à l'intérieur des villages ${ }^{28}$. Le processus d'endoctrinement et le système de punition étaient également centraux.

Circulations transnationales et adaptations locales de savoir-faire militaires

L'idée de construire des villages modèles pour regrouper la population rurale dans des territoires contestés n'est pas nouvelle et ne se limite pas à la période de la guerre froide. Il y a eu, par exemple, des missions créées -à partir du XVIIème siècle- par les jésuites en Amérique du Sud pour regrouper des populations autochtones ou des villages édifiés au début du XXème siècle dans le cadre du «welfare colonialism » en Afrique et en $\mathrm{Asie}^{29}$. Cependant, au début de la guerre froide, cette technique a évolué et a été associée aux techniques propres à la guerre contre-insurrectionnelle. Nous considérons que l'un des éléments les plus importants qui distinguent les villages stratégiques de contreinsurrection des autres expériences précédentes de regroupement de populations est la conjonction entre tactiques militaires et programmes dits de développement rural et de « modernisation » forcée.

Les armées françaises, britanniques et états-uniennes ont joué un rôle central dans la constitution de l'idée des villages stratégiques et dans sa diffusion transnationale. Finn Stepputat explore comment la Doctrine de la guerre révolutionnaire (DGR) - fondée notamment sur l'expérience de l'armée française en Indochine et en Algérie - a influencé d'abord l'armée américaine, puis les armées latino-américaines, comme celle du Guatemala. Élie Tenenbaum examine l'influence de l'armée britannique (notamment le plan Briggs et le travail de Rober G. K. Thompson en Malaisie) dans la conception du programme de Strategic Hamlets au Vietnam. Au Mozambique, étudié ici par João Paulo Borges Coelho, les Aldeamentos créés par le gouvernement colonial portugais se sont basés directement sur l'influence des armées françaises, britanniques et états-uniennes (notamment sur les expériences de la Malaisie et du Vietnam).

Qu'est-ce qu'implique, socialement et politiquement, la construction de villages stratégiques? Est-il possible de systématiser des éléments transversaux malgré les singularités de chaque cas ? Dans ce dossier, nous réunissons des enquêtes qui portent sur

${ }^{28}$ P. Colombo, « L'urbanisation forcée comme politique contre-insurrectionnelle. La vie au sein des villages stratégiques construits en Argentine (1976-1978) », art. cité.

${ }^{29}$ James Scott, Seeing like a State. How Certain Schemes to Improve the Human Condition Have Failed, New Haven, Yale University Press, 1998. 
la mise en place de ces programmes dans des contextes et des situations variés. Cependant, nous considérons que, malgré la singularité de chaque cas, les villages stratégiques ont en commun les caractéristiques suivantes :

- ayant pour cible principale la population civile, cette stratégie se trouve au cœur des programmes d'action civique militaire ;

- elle s'applique fréquemment aux marges de l'État ${ }^{30}$, c'est-à-dire sur des populations considérées comme marginales, et sur des espaces « marginaux »;

- les villages stratégiques font partie de projets ayant pour vocation à modifier l'ordre économique et politique de la région (la spécificité de ces changements variant selon les pays analysés) ;

- le programme implique toujours une reconfiguration territoriale beaucoup plus vaste que la seule création des villages, et qui vise à renforcer le contrôle du territoire et la présence de l'État dans la région. La «villagisation» donnerait ainsi lieu à une amélioration de la « lisibilité » du territoire et de la population ${ }^{31}$.

- avec des objectifs clairement contre-insurrectionnels, le programme vise à modifier les conditions sociales, politiques, économiques et spatiales qui empêcherait l'existence ou la réémergence des mouvements guérilleros.

Cette ébauche de systématisation a seulement la prétention d'offrir une grille de lecture pour aborder les discussions relatives à la circulation transnationale d'une technique de contrôle de population, tout en permettant d'explorer les particularités de chaque cas. De fait, il est important de souligner que, dans chaque situation, ces principes généraux ont été adaptés et modifiés. Le plus souvent, on ne retrouve pas nécessairement une traduction littérale de la doctrine contre-insurrectionnelle, mais plutôt des adaptations liées aux éléments suivants :

- la nature et la quantité des ressources à disposition de chaque armée (les ressources économiques varient mais aussi le type de géographie où les villages vont être édifiés) ;

- l'existence d'un lien direct entre la quantité de villages construits et la façon dont ils ont été bâtis. La magnitude du programme est très variable, qui va de réalisations très réduites (5 villages en Argentine avec environ 3000 personnes déplacées) à des projets titanesques (480 New Villages en Malaisie avec 572000 personnes $^{32}$ déplacées ou 2000 « centres de regroupement» en Algérie avec 2,5 millions d'habitants). Cependant, les números eux-mêmes sont sujets à controverse, comme le signale É. Tenenbaum en référence à la discussion sur le nombre de Strategic Hamlets effectivement construits au Vietnam ;

- les réinterprétations du programme à partir des histoires locales, liées à des politiques antérieures de relocalisation et de regroupement des populations. Dans ce sens, Neil MacMaster ${ }^{33}$ signale que le programme des «Mille Villages » mené par la France en

${ }^{30}$ Veena Das, Deborah Poole (eds), Anthropology in the Margins of the State, New Delhi, Oxford University Press, 2004.

${ }^{31}$ J. Scott, Seeing like a State. How Certain Schemes to Improve the Human Condition Have Failed, op. cit.

${ }^{32}$ K. Hack, « Malaya - Between Two Terrors: "People's History” and the Malayan Emergency », cité.

${ }^{33}$ Neil MacMaster, «From Tent to Village Regroupement: The Colonial State and Social Engineering of Rural Space, 1843-1962 », dans Ed Naylor (ed.), France's Modernising Mission. Citizenship, Welfare and the Ends of Empire, Portsmouth, Palgrave Macmillan, 2018, p. 109-131. 
Algérie a hérité non seulement des techniques contre-insurrectionnelles de l'armée, mais aussi de l'histoire de la colonisation des territoires « non civilisés » entamée en Algérie depuis 1843. Il insère donc les programmes de villages stratégiques dans la «longue durée » d'une Europe impérialiste ;

- le large éventail, quelquefois hétéroclite, d'expériences dont chaque armée s'est inspirée pour édifier les villages stratégiques. É. Tenenbaum explique par exemple que le programme de construction des Strategic Hamlets au Vietnam s'est inspiré des divers experiences, dès New Villages britanniques en Malaisie à des kibbutzim d'Israël ;

- les différentes versions de la mise en place nationale des programmes. F. Stepputat montre qu'au Guatemala il y a eu trois types de villages stratégiques : ceux de 1982 qui s'apparentaient à des camps de concentration, les aldeas modelo et le Polos de desarrollo de 1983-1984, et les communautés rurales créées durant la période post-conflit en 1990. Fabien Sacriste établit une distinction entre le programme des "Centres de regroupement» et celui des «Mille Villages» en Algérie, mais il signale aussi des différences à l'intérieur de chaque programme quant à son développement et aux ressources mises en place ;

- la possibilité que le sens du dispositif soit affecté aussi durant le déroulement du programme et qu'il change radicalement. Les villages stratégiques ne sont pas forcément des dispositifs « cohérents » ni « harmoniques », comme le signale J. P. Borges Coelho. $\mathrm{Au}$ moment de leur mise en place, les objectifs explicites de ces expérimentations d'ingénierie sociale peuvent se déformer et se transformer.

L'espace urbain comme instrument de guerre?

Dans son livre Outlaw Territories, Felicity Scott explore la manière dont le génocide et l'écocide sont reliés ${ }^{34}$. Pour l'armée, il ne s'agit pas uniquement d' "enlever l'eau au poisson ", il faut aussi modifier radicalement son environnement. Ces altérations font référence non seulement aux techniques dites de la "terre brûlée », mais aussi à un changement radical des conditions de vie de la population déplacée. Fort de sa participation aux guerres d'Indochine et d'Algérie, le colonel français Roger Trinquier a théorisé cette dynamique entre destruction et construction qui traverse les politiques de contre-insurrection : " À leur départ, les troupes d'intervention devront non seulement avoir détruit complètement les bandes, mais ne laisser derrière elles qu'une zone vidée de toutes ressources et parfaitement inhabitable $»^{35}$. S'il est nécessaire de rendre certains espaces inhabitables et d'autres habitables, c'est pour détruire l' " ennemi intérieur »" et supprimer les conditions de son émergence. Les manuels de contre-insurrection recommandent de construire de nouveaux espaces de vie (les villages stratégiques) et, parallèlement, de rendre inhabitables d'autres espaces en bouleversant les formes de vie antérieures.

34 Felicity D. Scott, Outlaw Territories. Environment of Insecurity/Architectures of Counterinsurgency, New York, Zone books, 2016.

${ }^{35}$ R. Trinquier, La guerre moderne, op. cit, p. 80.

${ }^{36}$ Mathieu Rigouste, "L'ennemi intérieur, de la guerre coloniale au contrôle sécuritaire », Cultures \& Conflits, 67, 2007, p. 157-174. 
L'idée de regrouper et d' « urbaniser » domine les programmes de villages stratégiques. Cela ne signifie pas que les militaires voulaient créer des villes, mais qu'ils souhaitaient plutôt bouleverser les modes de vie de la population rurale en introduisant certains éléments d'un mode de "vie urbain $»^{37}$. En dépit de l'échelle réduite de ces nouveaux espaces urbains, l'intention de l'État (réelle ou rhétorique) était bel et bien d'intégrer les paysans et ces territoires dans une structure relationnelle étendue des ? espaces urbains ${ }^{38}$. «L'urbanisation forcée» implique aussi l'idée d'une "modernisation forcée » des espaces ruraux. R. Thompson, ancien Secrétaire à la Défense en Malaisie et co-auteur du concept des New Villages $^{39}$, signale dans son manuel de contre-insurrection que l'objectif est que les villages assurent non seulement la protection, mais aussi la « prospérité » de la population : ils doivent être la porte d'entrée de la «vie moderne $»^{40}$. En analysant le cas des villages stratégiques du Vietnam, Nick Cullather signale qui pour le planificateur : « le village et le capitale se sont transformés en étapes [nécessaires] pour mettre en scène le spectacle de la modernité ${ }^{41}$. C'est l'espace « sauvage » qui subira le processus de "modernisation » contraint ${ }^{42}$. Michael Latham explique que, pour le gouvernement de John F. Keneddy, les actions anticommunistes devaient être accompagnées par des activités « pro-modernisation $»^{43}$.

Le fait que les espaces urbains soient devenus la cible délibérée des destructions dans des contextes de conflits armés a suscité une large réflexion théorique. La notion d'urbicide ${ }^{44}$ en particulier a permis d'explorer la façon dont la destruction des bâtiments, mais aussi des infrastructures urbaines a été utilisée comme un outil de guerre pour affaiblir les combattants et la population civile. Lutter contre l'infrastructure est aussi un combat mené contre la condition même d'existence et de reproduction de la communauté ${ }^{45}$. Cependant, les programmes militaires de constructions de villages stratégiques mis en œuvre en Amérique latine, en Asie et en Afrique démontrent que le pouvoir de « tuer des villes $\gg^{46}$ existe parallèlement à celui de créer des espaces urbains ex nihilo ${ }^{47}$. À ce titre, les politiques contre-insurrectionnelles ne luttent pas seulement contre la ville - entendue

${ }^{37}$ L'urbanité est donc ici principalement comprise comme un mode de vie. Edward Soja et Kanai, «The Urbanization of the World », dans Ricky Burdett, Deyan Sudjic (eds), The Endless City: The Urban Age Project by the London School of Economics and Deutsche Bank's Alfred Herrhausen Society, Londres, Pahidon, 2006, p. 54-68.

${ }^{38}$ F. Stepputat, « Politics of Displacement in Guatemala », art. cité.

${ }^{39}$ Voir l'article de É. Tenenbaum dans ce dossier.

${ }^{40} \mathrm{R}$. Thompson, Defeating Communist Insurgency: The Lessons of Malaya and Vietnam, op. cit., p. 133.

${ }^{41}$ Nick Cullather, «"The Target Is the People”: Representations of the Village in Modernization and National Security Doctrine ", dans Ryan Bishop, Gregory Clancey, John W. Phillips (eds), The City as Target, Abingdon, Routledge, 2012, p. 290.

${ }^{42}$ J. Scott, Seeing like a State. How Certain Schemes to Improve the Human Condition Have Failed, op. cit.

${ }^{43}$ Michael Latham, Modernization as Ideology: American Social Science and « Nation Building 》 in the Kennedy Era, Londres, University of North Carolina Press, 2000, p. 167.

${ }^{44}$ Stephen Graham, « Lessons in Urbicide », New Left Review, 19, 2003, p. 63-77.

${ }^{45}$ Martin Coward, Urbicide. The Politics of Urban Destruction, New York, Routledge, 2009 ; R. Bishop, G. Clancey, J. W. Phillips (eds), The City as Target, op. cit.

${ }^{46}$ S. Graham, Cities under Siege: The New Military Urbanism, Londres, New York, Verso, 2010.

${ }^{47}$ Le caractère urbain a souvent été associé à de vastes espaces et à de fortes densités de population. Cependant, selon certaines théories critiques urbaines, nous faisons face aujourd'hui à un " processus d'urbanisation étendue » dont l'urbanisme est principalement compris comme un mode de vie. Neil Brenner, «Theses on Urbanization », Public Culture, 25 (1), 2013, p. 85114). 
ici dans une acception culturelle et en tant que mode de vie - ou dans la ville, mais aussi par la ville.

Dans ce dossier, les auteurs analysent donc sous différents angles des programmes d'action civique militaire qui reprennent des idées de « modernisation » et d'urbanisation forcée, la place que ces idées occupent dans le corpus doctrinal des armées et la manière dont les objectifs de réorganisation territoriale et de contrôle de la population ont joué un rôle central dans de nombreux conflits armés du $\mathrm{XX}^{\mathrm{e}}$ siècle. Nous nous sommes également intéressés au fait que ces programmes militaires ont été conçus et présentés, dans une grande partie des cas, comme des programmes de « développement » (voir par exemple les articles de F. Stepputat, P. Colombo et J. P. Borges Coelho). Or que l'urbanisation apparaisse liée aux villages dans la grande partie des discours étatiques n'implique pas que, dans chaque cas, il y ait eu les ressources suffisantes pour monter l'infrastructure nécessaire à l'aboutissement du projet.

De plus, les termes mêmes de villages et d'urbanisation sont soumis à la critique par les auteurs de ce dossier. F. Sacriste discute en profondeur les limites du concept de village stratégiques, en proposant de parler de « rurbanisation » plutôt que d'urbanisation ${ }^{48}$ et de « villagisation »- pour faire référence à l'effort d'aménagement dont le degré est variable d'un lieu à l'autre (restant dans une grande partie des cas au niveau du camp) plutôt que de «village ». Il explore ainsi les différents usages des termes urbanisation et villages dans le cas de l'Algérie : la légitimité et la propagande (usages interne et externe), l'action publique, et un projet de développement autoritaire. Il entend le village plutôt comme un prétexte pour mener des opérations de guerre.

F. Stepputat expose que, dans le cas guatémaltèque, après une première phase de déplacement de populations chassées par les massacres, l'armée changera vers un modèle de construction de villages à partir d'une "image urbaine». Pour cet auteur, cette urbanisation comprend l'organisation en établissements groupés et centrés (les villages stratégiques), l'ordre administratif et spatial des habitations qui permet le gouvernement de la population à distance («l'ordre de la ville ») et, en dernier, la production de sujets. Pour le cas de l'Argentine durant la dictature militaire (1976-1983), P. Colombo signale que ces expériences d'urbanisation forcée sont fondées sur la croyance - présente dans les manuels militaires eux-mêmes - qu'en transformant des environnements de vie et de travail, les personnes qui habitent ces territoires changeront radicalement pour devenir des citoyens « civilisés », éloignés de l'influence de la guérilla.

Développement et sécurité nationale

La guerre, notamment après 1945, commence à être conçue comme un problème de développement, voire comme un projet scientifique ${ }^{49}$. C'est cet imbrication des mondes civil et militaire que l'étude des villages stratégiques permet d'explorer.

\footnotetext{
${ }^{48}$ Laurence Thomsin, «Un concept pour le décrire : l'espace rural rurbanisé », Ruralia, 9, 2001, pp.1-15.

${ }^{49}$ M. Latham, Modernization as Ideology: American Social Science and "Nation Building" in the Kennedy Era, op. cit., p. 153.
} 
En nous distanciant des discours qui associent le développement à une valorisation positive des stratégies dites de modernisation ${ }^{50}$, nous considérons qu'il est important d'aborder le développement de façon critique en essayant de comprendre ce qui est fait en son nom ${ }^{51}$ et en quoi il est utilisé comme une technique de gouvernement ${ }^{52}$. Durant la guerre froide, des programmes de « développement» financés par des institutions telles que la Fondation Ford ou l'USAID ont postulé que l' " antidote » contre le communisme se trouvait dans la modernisation des villages et le développement communal ${ }^{53}$. Les villages stratégiques de contre-insurrection semblent avoir repris ces mêmes principes, mais avec une finalité militaire particulière ${ }^{54}$. L'histoire du " village modèle » et son lien avec le « développement rural » ne devraient pas être considérés comme une simple et pragmatique stratégie de développement, mais plutôt comme une « technologie spatiale complexe et polyvalente ${ }^{55}$. À partir de l'analyse du village de Fuerte Esperanza en Argentine, P. Colombo explore la façon dont une stratégie contre-insurrectionnelle s'hybride avec un programme dit de "développement» pour reconfigurer de façon radicale un territoire conçu comme « indompté ». F. Stepputat signale le moment précis où l'armée guatémaltèque a commencé à introduire les thèses du développement dans sa doctrine : vers 1982 sont apparus des programmes de «pacification » : Fusiles y Frijoles ( $70 \%$ de haricots, $30 \%$ de balles ») et Techo, Trabajo y Tortilla (qui donnait un abri et de la nourriture en échange du travail accompli dans la zone du conflit), soutenus par l'USAID et le World Food Program. É. Tenenbaum souligne qu'au Vietnam l'USAID a joué aussi un rôle crucial en livrant des «Strategic Hamlets Kits » pour la construction des villages.

Un des objectifs centraux est de produire un changement social qui dissuadera les populations civiles de rejoindre la guérilla, notamment en changeant leurs conditions matérielles de vie. Concrètement, et dans une grande partie des cas, cette amélioration est restée au stade rhétorique de la propagande militaire. F. Sacriste démontre que la grande partie des «centres de regroupement» créés par le gouvernement français en Algérie étaient plutôt des camps de concentration. Dans cette même veine, Pierre Bourdieu et Abdelmalek Sayad ont insisté sur le fait que l' "action "humanitaire" " (les auteurs faisaient ici référence au travail de la SAS) menée en Algérie était « objectivement une arme de guerre, orientée vers le contrôle des populations ${ }^{56}$. Bien que les villages

\footnotetext{
${ }^{50}$ Pour une analyse des différentes récits sur le développement, voir Maria Stern, Joakim Öjendal, «Mapping the Security-Development Nexus: Conflits, Complexity, Cacophony, Convergence ? », Security Dialogue, 41 (1), 2010.

${ }^{51}$ Arturo Escobar, Ecountering Development: The Making and Unmaking of the Third World, Princeton, Princeton Univeristy Press, 1995; James Ferguson, The Anti-Politics Machine: «Development », Depolicization, and Burocratic Power in Lesotho, Minneapolis, University of Minnesota Press, 1994.

${ }^{52}$ Lars Buur, Steffen Jensen, Finn Stepputat, "The security-development nexus", dans L. Buur, S. Jensen, F. Stepputat (eds), The Security-Development Nexus. Epressions of Sovereignty and Securitization in Southern Africa, Cape twon, HSRC Press, 2007, p. 9-34.

${ }^{53}$ «Raffiné durant décennies, le «développement communautaire» était parmi les techniques de construction de la Nation les plus étudiées et imitées ». N. Cullather, " "The Target Is the People": Representations of the Village in Modernization and National Security Doctrine ", cité, p. 287.

54 Ibid.; F. D. Scott, Outlaw Territories. Environment of Insecurity/Architectures of Counterinsurgency, op. cit.

${ }^{55}$ Japhy Wilson, « Model Village in the Neoliberal Era: The Millennium Development Goals and the Colonization of Everyday Life », The Journal of Peasant Studies, 41 (1), 2014, p. 107-125.

${ }^{56}$ Pierre Bourdieu, Abdelmalek Sayad, Le déracinement. La crise de l'agriculture traditionnelle en Algérie, Paris, Les Éditions Minuit, 2014 (1964), p. 25.
} 
stratégiques aient pu être promus comme des espaces d'assistance et de protection militaire des villageois, Nicole Sackley signale qu'ils restent essentiellement et avant tout des instruments de guerre ${ }^{57}$.

J. P. Borges Coelho signale que les Aldeamentos du Mozambique se sont constitués comme des espaces concentrationnaires producteurs de violences. Et F. Sacriste rappelle que, dans le cas de l'Algérie, le déracinement a produit au moins deux types de violence : l'une, directe, a entraîné la destruction des villages, l'autre, plus indirecte, a produit, entre autres, la précarisation des conditions de vie et la surmortalité.

Biopolitique et pouvoir disciplinaire

Les villages stratégiques ont été conçus comme des « dispositifs disciplinaires ${ }^{58}$ dans lesquels les habitants devaient être recensés, identifiés, et enregistrés ${ }^{59}$. Dans certains cas, comme en Argentine ou au Guatemala, ils ont été aussi conçus comme des expériences sociales de développement contraint ${ }^{60}$ dans lesquelles les institutions et les services de la « vie moderne » devaient aider à créer un « nouveau citoyen » opposé à la guérilla ${ }^{61}$. Les études sur les villages stratégiques doivent donc également s'interroger sur les nouveaux types de citoyennetés auxquels les villages stratégiques ont donné naissance.

Ainsi, les villages stratégiques ont été pensés et construits comme des dispositifs non seulement disciplinaires, mais aussi sécuritaires ${ }^{62}$, permettant un fort contrôle de la population, tout en produisant des formes d'autocontrôle ${ }^{63}$. Analysant le cas des New Villages en Malaisie, Maureen Sioh explique qu'il s'agit de projets de contrôle étatique fondés sur la discipline et la politique morale du visuel, mais aussi sur une gouvernementalité qui implique le biopouvoir ${ }^{64}$. Selon elle, les New Villages « intègrent l'autorégulation de la majorité de la population, qui a commencé à voir son bien-être comme lié au sort de l'État $\rangle^{65}$.

Nous considérons que l'un des grands intérêts de l'étude des villages stratégiques est son potentiel d'exploration des projets mis en place par les États comme des sortes de microcosmes illustrant leur utopie totalitaire. Comme le signale Japhy Wilson, malgré la diversité des raisons idéologiques et géopolitiques qui ont présidé à la création des villages stratégiques, chaque cas peut être compris comme un projet utopique visant «à

${ }^{57}$ N. Sackley. "The village as Cold War sites: experts, development, and the history of rural reconstruction », art. cité, p. 483.

${ }^{58}$ Michel Foucault, Sécurité, territoire, population : cours au Collège de France, 1977-1978, Paris, Gallimard, 2004.

${ }^{59} \mathrm{~J}$. Tyner, War, Violence, and Population, op. cit.

${ }^{60}$ N. Sackley. "The village as Cold War sites: experts, development, and the history of rural reconstruction », , art. cité.

${ }^{61}$ P. Colombo, «L'urbanisation forcée comme politique contre-insurrectionnelle. La vie au sein des villages stratégiques construits en Argentine (1976-1978)», art. cité.

${ }^{62}$ M. Foucault, Sécurité, territoire, population : cours au Collège de France, 1977-1978, op. cit.

${ }^{63}$ Maureen Sioh, «Anxious Enactments: Postcolonial Anxieties and the Performance of Territorialization », Environment and Planning D: Society and Space, 28 (3), 2010, p. 467-486.

${ }^{64}$ Ibid.

${ }^{65}$ Ibidi., p.471. 
produire une société idéalisée incarnant une vision spécifique de l'ordre social $»^{66}$. Les villages stratégiques peuvent être considérés comme une clé de compréhension du projet constructif/biopolitique de ces gouvernements.

La population au centre du débat

Qui est l' « ennemi intérieur » pour l'armée ? Ce n'est pas seulement la guérilla ou les mouvements rebelles, mais aussi la population déplacée elle-même. Le rôle joué par les civils ne devait donc pas être laissé au hasard ${ }^{67}$. Partant notamment de ses expériences en Malaisie, R. Thompson a expliqué que la dispersion des habitants sur le territoire rendait le contrôle de la guérilla plus difficile. Pour s'assurer le "succès » militaire, les New Villages devaient "protéger la population", en assurant la bonne connexion entre villages, mais aussi en créant des milices, même si « l'aspect le plus vital de la protection, cependant, [restait] l'élimination dans le hameau de l'organisation clandestine des insurgés $\rangle^{68}$.

Bien que la population ait toujours été pour eux une source de suspicion, les militaires n'ont eu de cesse d'essayer de la « convertir » par un endoctrinement basé notamment sur la coercition physique, mais aussi sur des opérations de propagande ${ }^{69}$. Dans une grande partie des cas, la population déplacée a été forcée de se constituer en milices ou de « collaborer » d'une manière ou d'une autre avec les militaires.

Le village stratégique est censé devenir l'espace antiguérilla par excellence. La production du consensus va de pair avec la création d'une idée de communauté qu'encouragent les valeurs et symboles nationaux. Dans certains villages construits durant la dictature civilo-militaire en Argentine, la population déplacée était forcée, chaque jour, de hisser le drapeau et de chanter l'hymne national, d'aller à la messe et à des «réunions d'information" avec les militaires, de participer à des espaces « folkloriques » pour apprendre les danses nationales typiques... ${ }^{70}$ Des espaces et des temporalités étaient consacrés à la réinvention du citoyen argentin : un espace urbanisé et des citoyens « modèles » au service des politiques contre-insurrectionnelles. J. P. Borges Coelho signale que, dans le cas du Mozambique, les villages stratégiques avaient pour vocation principale de réorganiser le fonctionnement du pouvoir local rural.

Dans une grande partie des cas, les armées ont mené d'abord une étape de «nettoyage » (massacres, disparitions, assassinats...), puis une autre étape ayant pour but de « récupérer » la population civile. Selon F. Stepputat, l'armée guatémaltèque (suivant les principes de R. Trinquier) a conçu la population comme un combattant en plus. Elle a réussi à former des milices, patrullas civil de autodefensa (PAC), avec 900000 hommes. À cet égard, F. Stepputat rappelle que la population n'est pas toujours un sujet passif qui

${ }^{66}$ J. Wilson, « Model Village in the Neoliberal Era: The Millennium Development Goals and the Colonization of Everyday Life », art. cité., pp. 1-2.

${ }^{67}$ R. Trinquier, La guerre moderne, op. cit., p. 25.

${ }^{68} \mathrm{R}$. Thompson, Defeating Communist Insurgency: The Lessons of Malaya and Vietnam, op. cit., p. 124.

${ }^{69}$ H. Gurman, « Vietnam. Uprooting the Revolution: Counterinsurgency in Vietnam », dans H. Gurman (ed.), Hearts and Minds. A People's History of Counterinsurgency, op. cit.

${ }^{70} \mathrm{P}$. Colombo, «L'urbanisation forcée comme politique contre-insurrectionnelle. La vie au sein des villages stratégiques construits en Argentine (1976-1978)», art. cité. 
serait manipulé soit par la guérilla, soit par les militaires. Au Guatemala, durant la période de post-conflit, la population s'est servie des nouvelles infrastructures pour essayer de s'intégrer aux institutions étatiques émergeant avec les villages des années 1990.

Colonisation et expansion du capitalisme

En donnant aux armées régulières un accès privilégié à des territoires jusque-là sous contrôle de la guérilla, les villages stratégiques ont permis de modifier et d'augmenter le périmètre d'action de l'État. Dans certains pays, les programmes ont eu ainsi pour but d'achever des objectifs non militaires que les États avaient essayé d'accomplir auparavant sans succès, notamment, la « colonisation » de certaines régions et, par la même occasion, d'introduire le système capitaliste dans ces territoires ${ }^{71}$.

Les auteurs réunis dans ce dossier analysent différents types de réorganisation générés par la construction des villages stratégiques, et l'analyse du changement de système économique et politique de la région est sans doute centrale. Dans le cas du Chaco en Argentine, P. Colombo explore comment la dictature civilo-militaire a profité de l'usage de la force et de la mobilisation de toutes les « ressources » étatiques pour essayer de mettre en place un système économique fondé sur l'" efficacité » capitaliste de l'exploitation du territoire qui, dans d'autres conditions, aurait entraîné une forte résistance de la part de la population ${ }^{72}$. Au Guatemala, selon F. Stepputat, les déplacements et les relocalisations ont produit des profonds changements dans l'organisation et les représentations de l'espace, donnant lieu à l'inclusion de l'« espace abstrait » : l'espace adapté à l'administration capitaliste ${ }^{73}$.

Pour le débat que nous voulons mener ici, le cas de Mozambique se révèle très intéressant puisqu'il montre comment il est possible de détourner le modèle des villages stratégiques de sa mission contre-insurrectionnelle en le réutilisant dans le cadre d'un gouvernement socialiste. Curieusement, malgré l'échec militaire et humanitaire du programme durant la guerre de décolonisation, la plupart des aldeamentos ont survécu alors que le régime avait changé et que les objectifs économiques n'étaient plus les mêmes. L'une des raisons avancées par J. P. Borges Coelho pour expliquer la persistance du programme est que le gouvernement indépendant du FRELIMO devait, d'une part, consolider rapidement la structure du pouvoir, d'autre part, contrôler efficacement la société et le territoire. Par la suite, les villages stratégiques ont été le dispositif choisi pour mener un changement radical et rapide : 3000 aldeias comunais ont été construits sous le gouvernement du FRELIMO.

${ }^{71}$ Analysant des politiques de peuplement, Stéphanie Guyon et Gilles Pinson signalent que le village était promu comme une " "forme intermédiaire d'organisation sociale" et comme "facteur de progrès". (...) Une figure classique de la pensée coloniale ». Stéphanie Guyon, Gilles Pinson, «Introduction. Gouverner les populations par l'espace », Fabien Desage, Christelle Morel Journel, Valérie Sala Pala (dir.), Le peuplement comme politiques, Rennes, Presses universitaires de Rennes, 2014, p. 39.

${ }^{72}$ J. Scott, Seeing like a State. How Certain Schemes to Improve the Human Condition Have Failed, op. cit.

${ }^{73}$ F. Stepputat, « Politics of Displacement in Guatemala », art. cité, p. 74. 
Pacification ou quand la guerre change d'échelle

En introduisant les espaces de vie dans le calcul de la guerre, les villages stratégiques pose la question de l'échelle à laquelle celle-ci est pensée et mise en place. Avec le concept de «pacification » qu'ils définissent comme « le processus désignant l'ensemble des opérations militaires, politiques, idéologiques et le climat social qui alimentent un état intermédiaire (éventuellement décrit comme "transition") entre guerre et paix $\gg^{74}$, Michel Agier et Martin Lamotte soulignent que les principaux exemples se trouvent dans les périodes coloniales contre un « ennemi intime »à revoir : "L'ennemi désigné comme cible première de la pacification (...) est situé à la marge ou plus précisément à la frontière - au sens de la frontière de la conquête coloniale. Cette position "marginale" qui peut être le lieu de l'intimité (...) se reproduit presque à l'identique dans les différentes occurrences de la pacification $»^{75}$. Ces éléments préfigureraient les processus de pacification contemporains comme des guerres de proximité ${ }^{76}$. Les villages stratégiques peuvent ainsi être considérés comme des dispositifs spatiaux permettant d'introduire la sphère de l'intime dans le combat contre des mouvements révolutionnaires et, simultanément, de « faire la guerre » à partir d'une microéchelle. Ils permettent en outre d'approfondir l'analyse de ces zones grises d'indétermination dans lesquelles les techniques de gouvernement se situent entre guerre et paix. Certains auteurs considèrent que les villages stratégiques alimentent la situation de guerre déjà existante dans le pays (J. P. Borges Coelho), voire qu'ils peuvent être compris comme la continuation de la guerre par des autres moyennes (F. Stepputat).

$* * * *$

Face à la menace d'un « ennemi intérieur », certains États ont imaginé une solution qui s'est formalisée dans la modernisation de la vie paysanne, l'urbanisation des zones rurales et, surtout, la disparition du projet révolutionnaire à partir de la récupération des espaces « en rébellion ». Ce dossier de Critique internationale cherche à réunir pour la première fois des articles portant sur différents cas de construction de villages stratégiques à travers le monde, et ainsi à ouvrir un débat autour de la mise en place globale de cette stratégie militaire et de la façon dont elle a pu circuler au niveau transnational.

Pamela Colombo est docteure en sociologie de l'Université du Pays Basque (Bilbao) et du CSIC (Centre supérieur de la recherche scientifique espagnole, Madrid). Elle est actuellement chercheuse Marie Curie à l'IRIS (EHESS, Paris). Ses recherches portent sur les liens entre l'État, les politiques de contre-insurrection et les programmes de développement. Elle a organisé plusieurs colloques internationaux, dont les plus récents à Buenos Aires ( Ciudad y Dictadura », 2016), Paris («L'urbanisation comme instrument de guerre », 2016) et Montréal («Development Policies, Space and Violence in Latin America », 2017). Elle a publié, entre autres, Espacios de desparición. Vivir e

\footnotetext{
${ }^{74}$ Michel Agier, Martin Lamotte, «Les pacifications dans la ville contemporaine : ethnographies et anthropologie », L'Homme, 219-220, dossier Pacifications urbaines, 2016, p. 9.

${ }^{75}$ M. Agier, M. Lamotte, «Les pacifications dans la ville contemporaine : ethnographies et anthropologie $»$, art. cité, p. 10.

${ }^{76}$ Ibid., p. 11.
} 
imaginar los lugares de la violencia estatal (Tucumán, 1975-1983) (Buenos Aires, Miño y Dávila, 2017), codirigé l'ouvrage Space and the Memories of Violence. Landscapes of Erasure, Disappearance and Exception (Basingstoke, Palgrave Macmillan, 2014), et coordonné des numéros spéciaux dans les revues Human Remains and Violence, Cultures $\&$ Conflits, Clepsidra et Critique internationale.

pamela.colombo@ehess.fr 